\title{
Evaluation of Bone Marrow Findings of COVID-19 by Minimally Invasive Autopsies: A Single Tertiary Care Centre Experience from India
}

\author{
Abhishek Purohit ${ }^{1} \cdot$ Shruti Vaswani $^{1} \cdot$ Vikarn Vishwajeet $^{1} \cdot$ Deepak Kumar $^{2}$ (D) \\ Parag Vijayvergiya $^{2}$ - Swapnil Tripathi ${ }^{2}$ - Tanuj Kanchan ${ }^{3}$ - Nikhil Kothari ${ }^{4}$ • \\ Naveen Dutt ${ }^{5}$ Poonam Elhence ${ }^{1}$ Vijayalakshmi Nag ${ }^{6}$ Pradeep Bhatia ${ }^{4}$. \\ Mahendra K. Garg ${ }^{2} \cdot$ Sanjeev Misra ${ }^{7}$
}

Received: 25 April 2021 / Accepted: 11 September 2021 / Published online: 20 September 2021

(C) Indian Society of Hematology and Blood Transfusion 2021

\begin{abstract}
The 2019 novel coronavirus (2019-nCoV) originated in Wuhan City of China. In India, first confirmed case of coronavirus disease (COVID-19) was reported on January 30, 2020 and India is presently hit by second wave of COVID-19. The aim of the present study was to evaluate bone marrow findings of COVID-19 by minimally invasive autopsies to aid in understanding pathophysiology of the disease. This prospective study was conducted at tertiary
\end{abstract}

Presentation in a meeting: 61st Annual conference of Indian Society of Haematology and Blood transfusion (ISHBT) Haematocon virtual 2020.

Deepak Kumar

deepak1007sharma@gmail.com

Abhishek Purohit

purohitabhi80@gmail.com

Shruti Vaswani

vaswanipranjvas@gmail.com

Vikarn Vishwajeet

vikarn.pmch@gmail.com

Parag Vijayvergiya

paragvijay95@gmail.com

Swapnil Tripathi

dr.tripathi1994@gmail.com

Tanuj Kanchan

tanujkanchan@yahoo.co.in

Nikhil Kothari

kotharin@aiimsjodhpur.edu.in

Naveen Dutt

duttn@aiimsjodhpur.edu.in

Poonam Elhence

poonamabhay@gmail.com

Vijayalakshmi Nag

vijayalakshmi005@gmail.com care centre of Western Rajasthan. After obtaining approval from Institute's ethics committee and consent from next of kins, minimally invasive autopsies were conducted in 37 COVID-19 deceased patients within an hour after the death. The tissue specimens were processed with standard biosafety measures. Electronic medical records were reviewed retrospectively and patients' clinical details and results of laboratory investigations were noted. In this prospective study, bone marrow biopsies were collected from 37 COVID-19 minimally invasive autopsies. Mean age of these cases was 61.8 years and male: female ratio was 2.36. Comorbidities were observed in $25(67.5 \%)$ of all cases. Histopathological analysis revealed hypercellular,

Pradeep Bhatia

pk_bhatia@yahoo.com

Mahendra K. Garg

mkgargs@gmail.com

Sanjeev Misra

misralko@gmail.com

1 Department of Pathology, All India Institute of Medical Sciences, Jodhpur, Rajasthan, India

2 Department of Medicine, All India Institute of Medical Sciences, Jodhpur, Rajasthan, India

3 Department of Forensic Medicine and Toxicology, All India Institute of Medical Sciences, Jodhpur, Rajasthan, India

4 Department of Anaesthesia and Critical Care, All India Institute of Medical Sciences, Jodhpur, Rajasthan, India

5 Department of Pulmonary Medicine, All India Institute of Medical Sciences, Jodhpur, Rajasthan, India

6 Department of Microbiology, All India Institute of Medical Sciences, Jodhpur, Rajasthan, India

7 Department of Surgical Oncology, All India Institute of Medical Sciences (AIIMS), Jodhpur, Rajasthan, India 
normocellular and hypocellular marrow in 5,25 and 5 cases respectively (two biopsies were inadequate). There was marked interstitial prominence of histiocytes in 24 $(68.5 \%)$ cases. Out of these, evidence of haemophagocytosis was observed in 14 (40\%) cases, marked increase of haemosiderin laden macrophages in $20(57.1 \%)$ cases. There was prominence of plasma cells in $28(80 \%)$ cases. The present study attempted to fill the gap of dearth of literature from our country in COVID-19 autopsy studies by highlighting bone marrow findings. The data support the evidence of development of secondary haemophagocytic lymphocytosis in COVID-19 cases.

Keywords COVID-19 · Bone-marrow · Autopsy · Haemophagocytosis

\section{Introduction}

The 2019 novel coronavirus (2019-nCoV) or the Severe Acute Respiratory Syndrome Corona Virus-2 (SARS-CoV2), originated in Wuhan City of Hubei Province of China. In India, first confirmed case of coronavirus disease (COVID-19) was reported on January 30, 2020 and since then the virus has spread across the country [1]. More than 178 million cases of COVID-19 and more than 3.8 million deaths have been reported in the world [2,3]. Recently, a second wave had hit India and in the month of May, there was a time when more than four lakhs new cases and more than four thousand deaths were reported on a single day from India [3].

Patients commonly present with respiratory symptoms, however, symptoms related to other organ systems have also been reported [4]. Among many queries that need to be addressed are the mechanism(s) and pathophysiology underlying the development of these symptoms.

Historically, clinical autopsy has been an invaluable tool to understand the pathophysiology of diseases. Pathogen was novel and little was known about it, hence clinical autopsies were attempted in COVID-19 deaths as an aid to understand the biologic characteristics of SARS-CoV-2 and pathogenesis of COVID-19. Initial studies had focussed on lung, liver and heart by doing minimally invasive autopsies and reported diffuse alveolar damage with hyaline membrane in lungs as well as macro and microvascular thrombosis as a major histopathological finding in these patients [5-7]. Later, focus had shifted to study other organs as well including lymph nodes, spleen and bone marrow changes in COVID-19 deaths in an attempt to look for evidence of haemophagocytosis [8]. Recent studies noted high incidence of haemophagocytosis in bone marrow and provided data to support evidence of secondary haemophagocytic lymphohistiocytosis (HLH) in COVID19 patients $[9,10]$.

However, there is paucity of data on bone marrow findings in COVID-19 from India. In this study, we have made an effort to report bone marrow findings in thirtyseven autopsy specimens of COVID-19.

\section{Material and Methods}

This prospective study, which spanned from May 2020 to August 2020, was conducted at a tertiary care centre of Western Rajasthan. After obtaining approval from Institute's ethics committee and informed written consent from next of kins, minimally invasive autopsies were conducted on deceased COVID-19 patients within an hour of the death. All of these patients were diagnosed COVID-19 cases admitted in our Institution and had succumbed to the disease. These cases were diagnosed at the time of admission by polymerase chain reaction (PCR) test for SARS-CoV-2 performed on a nasopharyngeal swab and were managed in accordance with guidelines published by Ministry of Health \& Family Welfare (MoHFW) and Institutional protocol [11]. Bone marrow biopsy specimens were retrieved from posterior superior iliac spine and were kept in neutral buffered formalin for $48 \mathrm{~h}$ and then processed with standard biosafety measures.

Formalin-fixed paraffin embedded tissues were sectioned on glass slides and stained with Haematoxylin and Eosin (H\&E). The bone marrow biopsies were evaluated for cellularity, overall appearance of haematopoietic components and to look for presence of haemophagocytosis by three pathologists independently. Immunohistochemical staining was performed for CD68 (Thermo Fisher Scientific, KP-1, 1:400) and CD138 (Thermo Fisher Scientific, MI15, ready to use) on bone marrow biopsy sections to highlight macrophages and plasma cells respectively.

We adopted visual estimation of plasma cell percentage subjectively. H\&E sections and CD138 sections were examined by two pathologists independently to look for interstitial prominence and/or cluster of plasma cells. Plasma cells were considered increased when there was either interstitial prominence of plasma cells or if there were presence of clusters of plasma cells in the bone marrow biopsy sections.

Electronic medical records of all cases were reviewed retrospectively and patients' demographic data, clinical details including presence of co-morbidities and results of laboratory investigations [including complete blood count (CBC), X-ray chest, D-dimer, Serum interleukin-6 (IL-6) levels and liver function tests (LFT)] were noted.

Statistical analysis was performed using SPSS (version 20.0, Armonk, NYN:IBM corp). Continuous variables 
were expressed in mean $\pm \mathrm{SD}$ and categorical variables were expressed in number $(\%)$. Independent student ' $t$ ' test and Chi square test were applied to calculate $p$-value for continuous data and categorical variables respectively. $p$ value less than 0.05 was considered as statistically significant.

\section{Results}

In this prospective study, a total of 37 bone marrow biopsies were done while performing minimally invasive clinical autopsies during the study duration.

Mean age of the cases was 61.8 years (range, 28-85 years) and male:female ratio was 2.36:1. Comorbidities were observed in $25(67.5 \%)$ cases (Table 1). It was observed that $14(40 \%)$ cases had mild anaemia whereas severe anaemia, leucopenia and thrombocytopenia were observed in one case each. Bicytopenia was noted in three $(8.3 \%)$ cases and pancytopenia was observed in two $(5.5 \%)$ cases. On CBC, $13(36.1 \%)$ cases did not show any cytopenia.

Post-mortem histopathological analysis of bone marrow sections revealed normocellular marrow in 25 (71.4\%) cases and hypercellular marrow in five (14.3\%) patients whereas bone marrow was hypocellular marrow in five

Table 1 Basic clinical data of COVID-19 suspect and positive cases

\begin{tabular}{ll}
\hline Characteristics & Result \\
\hline Age, (Mean \pm 2SD) (years) & $62.17 \pm 11.20$ \\
Male, no. (\%) & $28(75.67)$ \\
Female, no. (\%) & $9(24.3)$ \\
Comorbidities, no. (\%) & $15(40.54)$ \\
Diabetes mellitus, type 2 & $12(32.43)$ \\
Hypertension & $04(10.8)$ \\
Coronary artery disease & $02(5.4)$ \\
Interstitial lung disease & $02(5.4)$ \\
Hyperthyroidism & $01(2.7)$ \\
Malignancy & $01(2.7)$ \\
Bronchial Asthma & $04(10.8)$ \\
Others & $12(32.43)$ \\
None & \\
Chest X-ray findings, no. (\%) & $03(8.1)$ \\
Normal & $08(21.62)$ \\
Unilateral lung opacities & $26(70.2)$ \\
Bilateral lung opacities &
\end{tabular}

SD: standard deviation

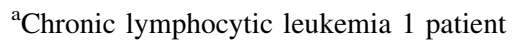

${ }^{\mathrm{b}}$ Nocardiosis 1 patient, Tuberculosis 1 patient, Rheumatic heart disease 1 patient, Parkinson's 1 patient
(14.3\%) cases (Two biopsies were inadequate for conclusive opinion). Bone marrow biopsies revealed presence of all haematopoietic components. Granulocytic series revealed mild prominence with shift to left in all the cases and marked prominence of megakaryocytes was noted in one $(2.7 \%)$ case. In $24(68.5 \%)$ cases, there was marked interstitial prominence of histiocytes scattered throughout the sections. Histiocytes revealed evidence of haemophagocytosis (Fig. 1) in $14(40 \%$ ) cases and there was marked increase of haemosiderin laden macrophages in $20(57.1 \%)$ cases. In two cases, the haemophagocytic histiocytes formed aggregates. On H\&E and CD138 stained sections, prominence of plasma cells was noted (Fig. 1) in $28(80 \%)$ cases. It was noted as interstitial increase in $78.6 \%(22 / 28)$ and as small aggregates in $21.4 \%$ $(6 / 28)$ cases. Immunohistochemical staining with antiCD68 (Fig. 2) and anti-CD138 antibodies (Fig. 3) highlighted prominence of histiocytes and plasma cells respectively. There was no evidence of necrosis, granuloma formation or presence of any haemoparasite in any of the bone marrow biopsy.

We divided cases in to two groups based on presence or absence of haemophagocytosis on bone marrow examination (Group A and B respectively). Table 2 reveals comparison of various parameters (Including temperature at the time of admission, age, haemoglobin, Total leucocyte count, platelet count, Neutrophil-Lymphocyte ratio (NLR), D-dimer, IL-6, aspartate aminotransaminase (AST), alanine aminotransferase (ALT), alkaline phosphatase (ALP), total bilirubin and duration of hospital stay) between these two groups. We observed that there was statistically significant difference in respect to NLR and duration of hospital stay between two groups (Table 2).

\section{Discussion}

Clinical autopsy has an important place in human history in understanding pathobiology of various diseases. Although many studies have been published in an attempt to understand the disease pathobiology by conducting clinical autopsies in COVID-19 deaths in West, literature from Indian subcontinent is scarce. Prominent observations have been damage to the surface layers of airway epithelial cells and massive lung involvement with diffuse alveolar damage (DAD) and microvascular thrombi ${ }^{5}$. With time, focus has moved towards study of other organ systems and immune dysfunction and development of hyperinflammatory state have been implicated for morbidity and mortality of affected patients. Studies have also observed an overproduction of cytokines, possibly correlating with secondary HLH [12]. Hence, evaluation of bone marrow in 


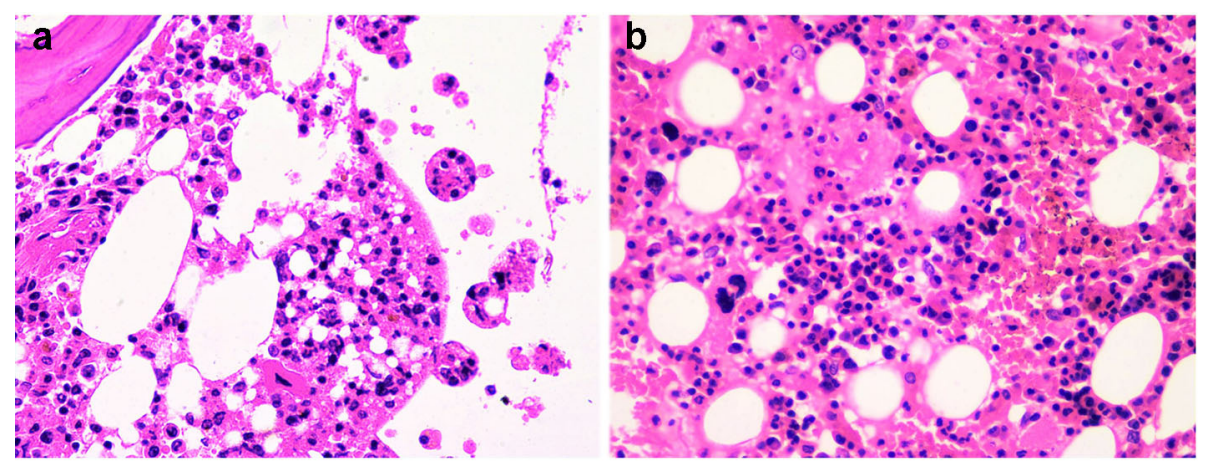

Fig. 1 Sections from bone marrow biopsy. a Section reveal myeloid and erythroid components along with paratrabecular prominence of histiocytes with evidence of haemophagocytosis b Section reveals all haematopoietic components with interstitial prominence of plasma cells (Haematoxylin and Eosin; 40X)

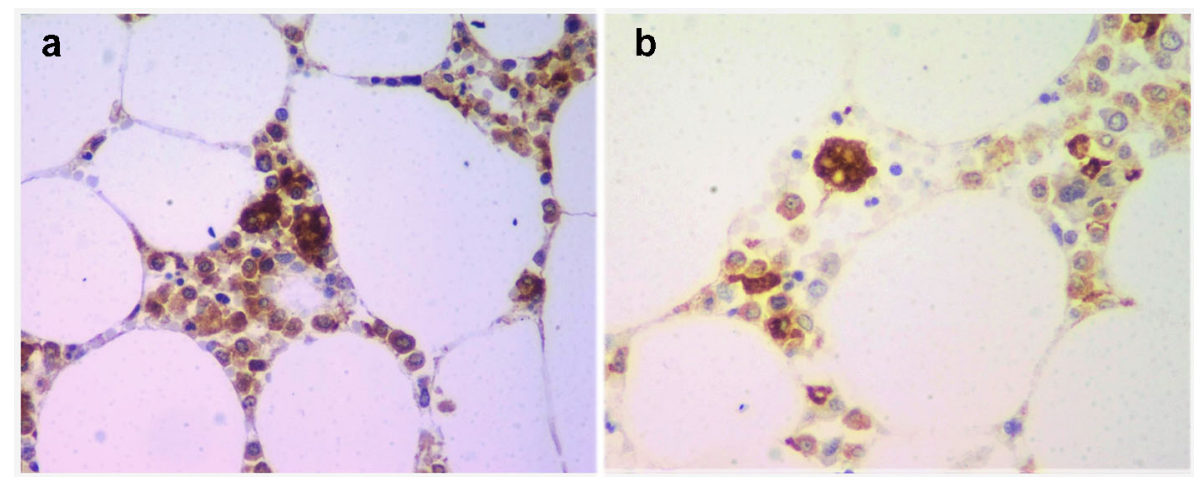

Fig. 2 Immunohistochemical staining for CD68 on bone marrow biopsy section highlights histiocytes with some showing evidence of haemophagocytosis (a and b; 40X)
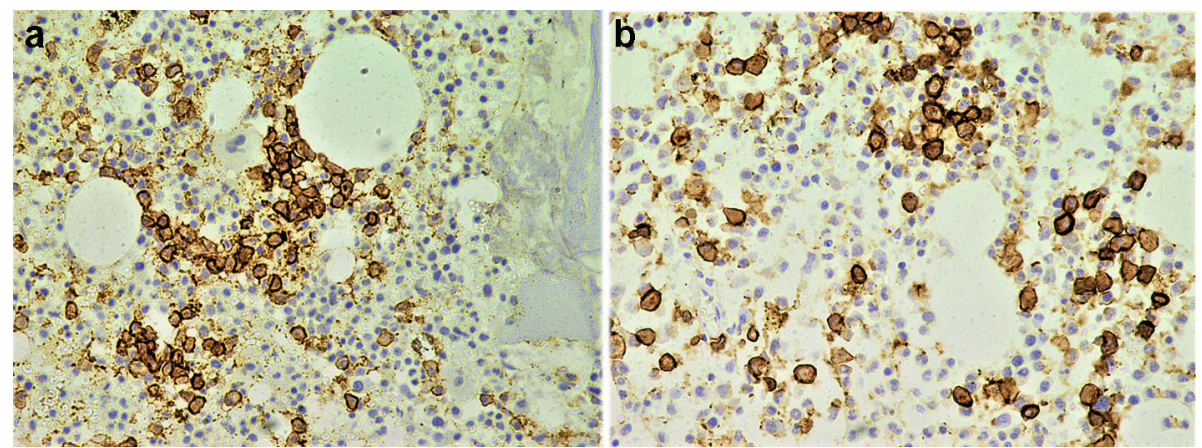

Fig. 3 Immunohistochemical staining for CD138 on bone marrow biopsy section highlights plasma cells (a and b; 40X)

affected patients is important for better understanding pathophysiology.

There is dearth of literature from Indian subcontinent and in the present study, we made an attempt to fill this gap by evaluating bone marrow histopathology in clinical autopsy specimens from COVID-19 patients.
Studies from West including United states of America, Spain and United Kingdom have observed trilineage haematopoiesis, myeloid prominence, histiocytic prominence and evidence of haemophagocytosis prominently $[10,13,14]$.

In addition, Prieto-Pérez et al. also noted prominence of plasma cells, aggregates of CD8 + lymphocytes and 
Table 2 Comparative clinical and laboratory data of COVID-19 cases

\begin{tabular}{|c|c|c|c|c|}
\hline & $\begin{array}{l}\text { All cases (35) } \\
(\text { Mean } \pm 2 \mathrm{SD})\end{array}$ & $\begin{array}{l}\text { Group } A^{a}(25) \\
(\text { Mean } \pm 2 S D)\end{array}$ & $\begin{array}{l}\text { Group } B^{b}(10) \\
(\text { Mean } \pm 2 S D)\end{array}$ & $p$-value \\
\hline Temperature at the time of admission $\left({ }^{\circ} \mathrm{C}\right)$ & $100.026 \pm 1.273$ & $100.24 \pm 1.082$ & $99.49 \pm 1.6$ & 0.197 \\
\hline Age (Years) & $62.17 \pm 11.20$ & $60.36 \pm 11.58$ & $66.7 \pm 9.19$ & 0.103 \\
\hline $\mathrm{Hb}(\mathrm{gm} / \mathrm{dL})$ & $12.05 \pm 1.93$ & $12.05 \pm 2.15$ & $12.06 \pm 1.38$ & 0.998 \\
\hline $\operatorname{TLC}\left(\times 10^{3} /\right.$ cumm $)$ & $11.42 \pm 15.55$ & $8.43 \pm 4.233$ & $18.58 \pm 27.57$ & 0.276 \\
\hline NLR & $10.03 \pm 9.14$ & $14.17 \pm 12.01$ & $6.92 \pm 5.18$ & 0.017 \\
\hline Platelet count $\left(\times 10^{3} /\right.$ cumm $)$ & $222.73 \pm 87.63$ & $212.5 \pm 80.40$ & $247.3 \pm 103.4$ & 0.358 \\
\hline D-Dimer (microgram/mL) & $3.94 \pm 2.99$ & $3.52 \pm 2.326$ & $5.44 \pm 4.63$ & 0.365 \\
\hline IL-6 (pg/mL) & $423.61 \pm 454.34$ & $401.44 \pm 463.54$ & $467.94 \pm 459.31$ & 0.728 \\
\hline AST (U/L) & $72.92 \pm 48.85$ & $61.0 \pm 31.39$ & $106.03 \pm 72.19$ & 0.103 \\
\hline ALT (U/L) & $46.25 \pm 32.70$ & $44.07 \pm 30.67$ & $52.31 \pm 39.14$ & 0.579 \\
\hline ALP (IU/L) & $136.50 \pm 142.17$ & $124.76 \pm 99.20$ & $169.11 \pm 228.54$ & 0.587 \\
\hline Total bilirubin $(\mu \mathrm{mol} / \mathrm{L})$ & $15.59 \pm 20.56$ & $16.14 \pm 23.42$ & $14.06 \pm 9.75$ & 0.719 \\
\hline Duration of hospital stay (days) & $9.54 \pm 6.89$ & $10.80 \pm 7.3$ & $6.40 \pm 4.67$ & 0.044 \\
\hline
\end{tabular}

${ }^{\mathrm{a}}$ Group A-Cases wherein bone marrow examination revealed histiocytosis and evidence of haemophagocytosis

${ }^{\mathrm{b}}$ Group B-Cases wherein bone marrow examination did not reveal histiocytosis and evidence of haemophagocytosis,

Hb: Haemoglobin; TLC: Total Leucocyte Count; NLR: Neutrophil-Lymphocyte ratio; IL-6: Interleukin-6; AST: Aspartate Aminotransferase; ALT: Alanine Aminotransferase; ALP: Alkaline Phosphatase

frequent presence of clusters of megakaryocytes. Further, they also performed bone marrow biopsy in three live COVID-19 patients and found bone marrow haemophagocytosis as well as other criteria of making a diagnosis of secondary HLH [13]. In our study, we did not find lymphoid aggregates and the bicytopenia or pancytopenia was also not a frequent finding.

In an Italian study, bone marrow biopsies were examined in 22 COVID-19 autopsies which revealed prominence of macrophages, haemophagocytosis and hypocellular bone-marrow and megakaryocytic hyperplasia whereas we observed hypocellular bone marrow in just five cases and megakaryocytic hyperplasia in one case only [15].

Bryce et al., in their study of 67 cases from New York city, could study bone marrow in six cases and found haemophagocytosis in four cases and decreased megakaryocytes in one case. In addition, they studied splenic tissues inn 22 cases and noted haemophagocytic histiocytes in seven cases [16]. Literature has emphasized that NLR has good predictive values not only on disease severity but also on mortality in patients with COVID-19 infection. Our findings are in accordance with the published literature [17].

Frequent observations of haemophagocytosis in previous studies as well as in our study support the hypothesis that HLH is induced due to COVID-19 by virtue of activation of the IL-1/IL-6 pathway, including overproduction of IL- $1 \beta$ by macrophages. In our study we noted that IL-6 levels were increased in all the cases, however, it was increased in all the cases irrespective of presence of haemophagocytosis.

Evidence of bone-marrow haemophagocytosis is just one criteria and is not sufficient to make a diagnosis of secondary HLH in these COVID-19 deaths, as haemophagocytosis may be secondary to infection-induced processes in the body. In past, studies have shown that the presence of marrow haemophagocytosis has limited sensitivity $(83 \%)$ and specificity $(60 \%)$ for the diagnosis of HLH and should be supplemented by other criteria [18].

The present study highlights the bone marrow changes, which are primarily reactive in nature and showed haemophagocytosis in majority of cases. While the major thrust is on lungs in COVID-19, changes observed in present study might be result of an indirect insult to the bone-marrow milieu in COVID-19 infections.

To conclude, the present study attempts to fill the gap of dearth of literature from our country in COVID-19 autopsy studies by highlighting bone marrow findings. We identified high incidence of haemophagocytic histiocytes in patients with fatal COVID-19. Although we could not do complete work-up for sHLH still, our findings suggest that a differential diagnosis of sHLH should be kept high in severe and deteriorating cases of COVID-19 and ancillary investigations must be done.

Acknowledgements We acknowledge the efforts of all the health care workers in this project. 
Authors' Contributions AP: Conceptualization, Investigation, Resources, Data curation, Writing-Original draft preparation. SV: Investigation, Writing- Original draft preparation. VV: Investigation, Resources, Writing- Original draft preparation. DK: Investigation, Resources, Writing- Original draft preparation. PV: Data Curation, Writing-Original Draft. ST: Data Curation, Writing-Original Draft. TK: Data Curation, Writing - Original Draft. NK: Data Curation, Writing - Original Draft. ND: Data Curation, Writing-Original Draft. PE: Conceptualization, Methodology, Writing-Review and Editing, Supervision. VN: Conceptualization, Methodology. PB: Conceptualization, Methodology. MK: Conceptualization, Methodology, Writing-Review and Editing, Supervision. SM: Conceptualization, Methodology.

Funding Nil.

Data Availability Yes.

\section{Declarations}

Conflict of interest Nil.

\section{Consent for Publication Nil.}

Ethical Approval The study was approved by Institute's Ethics Committee (AIIMS/IEC/2020-21/3058).

\section{References}

1. Singhal T (2020) A review of coronavirus disease-2019 (COVID19). Indian J Pediatr 87(4):281-286. https://doi.org/10.1007/ s12098-020-03263-6

2. World Health Organization. Situation reports. Available at: https://www.who.int/emergencies/diseases/novel-coronavirus2019/situation-reports/. Accessed 20 April 2021.

3. Coronavirus Outbreak. Available at: https://www.worldometers. info/coronavirus/. Accessed $19^{\text {th }}$ June 2021.

4. Singhania N, Bansal S, Singhania G (2020) An atypical presentation of novel coronavirus disease 2019 (COVID-19). Am J Med 133(7):365-366. https://doi.org/10.1016/j.amjme.2020.03.026

5. Xu Z, Shi L, Wang Y et al (2020) Pathological findings of COVID-19 associated with acute respiratory distress syndrome. Lancet Respir Med 8:420-422

6. Dolhnikoff M, Duarte-Neto AN, de Almeida Monteiro RA et al (2020) Pathological evidence of pulmonary thrombotic phenomena in severe COVID-19. J Thromb Haemost 18:1517-1519

7. Fox SE, Akmatbekov A, Harbert JL, Li G, Quincy Brown J, Vander Heide RS (2020) Pulmonary and cardiac pathology in African American patients with COVID-19: an autopsy series from New Orleans. Lancet Respir Med 8(7):681-686. https://doi. org/10.1016/S2213-2600(20)30243-5

8. Prilutskiy A, Kritselis M, Shevtsov A, Yambayev I, Vadlamudi C, Zhao Q, Kataria Y, Sarosiek SR, Lerner A, Sloan JM, Quillen K (2020) SARS-CoV-2 Infection-associated hemophagocytic lymphohistiocytosis: an autopsy series with clinical and laboratory correlation. Am J Clin Pathol 154(4):466-474. https://doi. org/10.1101/2020.05.07.20094888

9. Núñez-Torrón C, Ferrer-Gómez A, Moreno EM, Pérez-Mies B, Villarrubia J, Chamorro S, López-Jiménez J, Palacios J, PirisVillaespesa M, García-Cosío M (2021) Secondary haemophagocytic lymphohistiocytosis in COVID-19: correlation of the autopsy findings of bone marrow haemophagocytosis with HScore. J Clin Pathol. https://doi.org/10.1136/jclinpath-2020207337

10. Harris CK, Hung YP, Nielsen GP, Stone JR, Ferry JA (2021) Bone marrow and peripheral blood findings in patients infected by SARS-CoV-2. Am J Clin Pathol 155(5):627-637. https://doi. org/10.1093/ajcp/aqaa274

11. MoHFW I Home. Accessed August 28th, 2020. https://www. mohfw.gov.in

12. Felsenstein S, Herbert JA, McNamara PS, Hedrich CM (2020) COVID-19: Immunology and treatment options. Clin Immunol 215:108448. https://doi.org/10.1016/j.clim.2020.108448

13. Prieto-Pérez L, Fortes J, Soto C et al (2020) Histiocytic hyperplasia with hemophagocytosis and acute alveolar damage in COVID-19 infection. Mod Pathol 33:2139-2146. https://doi.org/ 10.1038/s41379-020-0613-1

14. Mehta P, McAuley DF, Brown M et al HLH Across Speciality Collaboration, UK (2020) COVID-19: consider cytokine storm syndromes and immunosuppression. Lancet 395:1033-1034

15. Falasca L, Nardacci R, Colombo D, Lalle E, Di Caro A, Nicastri E et al (2020) Postmortem findings in Italian patients with COVID-19: a descriptive full autopsy study of cases with and without comorbidities. J Infect Dis 222(11):1807-1815

16. Bryce C, Grimes Z, Pujadas E, Ahuja S, Beasley MB, Albrecht R et al (2020) Pathophysiology of SARS-CoV-2: targeting of endothelial cells renders a complex disease with thrombotic microangiopathy and aberrant immune response. The Mount Sinai COVID-19 autopsy experience. MedRxiv. https://doi.org/ 10.1101/2020.05.18.20099960

17. Li X, Liu C, Mao Z et al (2020) Predictive values of neutrophilto-lymphocyte ratio on disease severity and mortality in COVID19 patients: a systematic review and meta-analysis. Crit Care 24:647. https://doi.org/10.1186/s13054-020-03374-8

18. Goel S, Polski JM, Imran H (2012) Sensitivity and specificity of bone marrow hemophagocytosis in hemophagocytic lymphohistiocytosis. Ann Clin Lab Sci 42:21-25 (PMID: 22371906)

Publisher's Note Springer Nature remains neutral with regard to jurisdictional claims in published maps and institutional affiliations. 